\title{
The nodule-specific multifunctional channel NOD26 of Medicago truncatula is crucial for nitrogen-fixing symbiosis
}

\section{Romina Frare}

INTA: Instituto Nacional de Tecnologia Agropecuaria

\section{Cristina Gómez}

INTA: Instituto Nacional de Tecnologia Agropecuaria

\section{María Paula López-Fernández}

INTA: Instituto Nacional de Tecnologia Agropecuaria

\section{Karina Alleva}

INTA: Instituto Nacional de Tecnologia Agropecuaria

\section{Pablo Nikel}

Technical University of Denmark Center for Biological Sequence Analysis

\section{Nicolás Ayub}

INTA: Instituto Nacional de Tecnologia Agropecuaria

Gabriela Soto ( $\nabla$ soto.gabrielacinthia@inta.gob.ar)

CONICET https://orcid.org/0000-0002-3492-1268

\section{Research Article}

Keywords: symbiosis, legume, rhizobia, water, ammonia, nodulin-26

Posted Date: February 17th, 2021

DOl: https://doi.org/10.21203/rs.3.rs-171869/v1

License: (a) This work is licensed under a Creative Commons Attribution 4.0 International License. Read Full License 


\section{Abstract}

The multifunctional channel NOD26, identified and extensively studied (both biochemically and biophysically) in soybean, is a major protein component of the symbiosome membrane. The water and ammonia transport activities of NOD26 are thought to be important for nodule development, osmotic balance, and ammonia efflux from the symbiosome. However, the widely accepted relevance of NOD26 in nitrogen-fixing symbiosis has never been explored in planta. Recently, we have reported the emergence of NOD26 in the nitrogen-fixing clade of angiosperms via tandem duplication. Here, we characterized the two copies of NOD26 from Medicago truncatula (Medtr8g087710 and Medtr8g087720) in their transport abilities, and at gene expression and genetic levels. Similar to their homologous soybean gene, MtNOD26 genes encode water and ammonia transport activities in heterologous expression systems. By using multiple transcriptional studies (RT-qPCR, transcriptional fusion and RNA-Seq analyses), we found that the expression of MtNOD26 copies is restricted to the nodule and gradually increases from the bacteriafree meristematic region to the nitrogen-fixation zone. Under nitrogen-limiting soil conditions, the homozygous insertional mutant lines of these two MtNOD26 genes had the same aberrant nodulation phenotype and chlorosis. Similar to uninoculated wild-type plants, inoculated mutants were unable to grow in minimal medium without a nitrogen source. Collectively, our findings suggest functional equivalence between MtNOD26 copies and underline a crucial role of NOD26 in symbiotic nitrogen fixation.

\section{Summary}

Nitrogen is an essential macronutrient required for plant growth and development. However, its bioavailability in natural environments is normally limited. Under this strong selective pressure, some plant species belonging to the monophyletic nitrogen-fixing clade of angiosperms have established a symbiosis with nitrogen-fixing bacteria (e.g. rhizobia). These plants provide a carbon energy source, generally in the form of a dicarboxylate, utilized by their microsymbionts to produce adenosine triphosphate (ATP) in support of nitrogen fixation, and obtain ammonia as the principal beneficial compound (diCenzo, et al. 2020). Within the nitrogen-fixing clade, legumes stand out due to their extraordinary diversity, ecological success and agronomic relevance. In legume-rhizobia symbioses, rhizobia infect and occupy infected plant cells within a particular plant structure known as the nodule. Nitrogen-fixing ammonia-excreting forms of rhizobia, commonly known as bacteroids, reside within organelle-like structures called symbiosomes (Jones, et al. 2007). During the release of rhizobia into the plant host cytoplasm, the symbiosome membrane (SM) forms an uninterrupted membrane around each bacterium and delineates a new space between the SM and the bacterial outer membrane, named the symbiosome space. The acidic $\mathrm{pH}$ in the symbiosome space, which is a consequence of proton pumping by both the SM ATPase and the bacteroid respiratory chain, results in the conversion of ammonia to ammonium in this space, facilitating the efficient removal of fixed nitrogen from the bacteroid (Blumwald, et al. 1985; Pierre, et al. 2013). 
Since the symbiosome represents the main organelle within these non-vacuolated infected plant cells and the SM osmotic water permeability is higher than normal membrane bilayer diffusion rates, the symbiosome can contribute to the regulation of the cell volume during nitrogen-fixing symbiosis (Routray, et al. 2015). Besides its osmoregulatory function in infected plant cells, the SM controls the movement of metabolites between the two symbiotic partners, including the transfer of ammonia from the bacteroid to the plant cytoplasm. To play multiple and complex roles, the SM contains a set of channels and transporters which are specifically induced during nodule formation (Mergaert, et al. 2020). These types of plant-encoded, nodule-specific proteins are normally known as nodulins.

Among nodulins, there is a $26-\mathrm{kDa}$ membrane-intrinsic protein (nodulin-26 or NOD26), a member of the aquaporin (AQP) superfamily, which facilities the transport of water and small solutes across cell membranes in a passive manner (Perez Di Giorgio, et al. 2014). NOD26, firstly identified in soybean plants, is a major protein component (10\%) of the SM (Fortin, et al. 1987). NOD26 is exclusively localized in the SM, and is used as a marker of this specific membrane in studies of subcellular localization of proteins inside nodules (Clarke, et al. 2015). To understand the possible functions of NOD26 in nitrogenfixing symbiosis, its abundance has been analyzed at protein level by using different anti-NOD26 antibodies and its transport activities have been studied by using the native NOD26 and different heterologous expression systems.

NOD26 seems to be an essential component of nitrogen-fixing nodules because its synthesis is induced during nodule organogenesis independently of the development of the symbiosome (Fortin, et al. 1987). The initial biochemical characterization of NOD26 indicated that this channel is a substrate for $\mathrm{Ca}^{2+}$ dependent phosphorylation (Weaver, et al. 1991). In regulatory terms, NOD26 protein synthesis increases gradually during nodule development under normal physiological conditions, where the phosphorylation of NOD26 coincides with the establishment of mature nitrogen-fixing symbiosomes (Guenther, et al. 2003). Remarkably, NOD26 phosphorylation is enhanced by water deprivation conditions and salinity, providing additional evidences that NOD26 is regulated at post-transcriptional level and suggesting that NOD26 can be part of the adaptive responses of infected cells to osmotic stress (Guenther, et al. 2003). Regarding transport activities, NOD26 is a multifunctional channel that facilitates the bidirectional transport of water and ammonia, as well as probably of other small solutes such as glycerol and formamide, across cell membranes in a passive manner, with ammonia being the preferred substrate (Dean, et al. 1999; Hwang, et al. 2010; Rivers, et al. 1997). The phosphorylation of NOD26 stimulates its water transport rate and probably modulates its primary selectivity (Guenther, et al. 2003; Niemietz and Tyerman 2000), suggesting that the post-transcriptional regulation of NOD26 plays a central role in its multifunctional nature. In addition, the C-terminal domain of NOD26 can act as a docking site for glutamine synthetase in the infected cell cytoplasm (Masalkar, et al. 2010), probably facilitating the rapid assimilation of the transported ammonia and preventing the toxic accumulation of ammonia in the cytoplasm. The detection of large amounts of both NOD26 and cytosolic glutamine synthetase in the SM proteome (Clarke, et al. 2015) provides additional evidences supporting the crucial role of NOD26 in the release of ammonia from the symbiosome. 
By integrating genomic, phylogenetic, transcriptomic and metabolic analyses, we have recently shown that NOD26 emerged in the nitrogen-fixation clade of angiosperms by tandem duplication (Frare, et al. 2018), probably from an ancestral AQP specifically induced in root cells during anoxic stress, such as lactate channel AtNIP2;1 (Beamer, et al. 2020). Contrary to other legumes (e.g. soybean and common bean), Medicago truncatula would have two active copies of NOD26 (Medtr8g087710=NOD26a and Medtr8g087720=NOD26b) (Fig. 1a), a fact that probably reflects an ancestral feature of nitrogen-fixing species (Fig. 1b-c) (Frare, et al. 2018). Thus, the experimental study of these genes individually can not only show their current function in $M$. truncatula but also recapitulate their importance in ancestral nitrogen-fixing plant species. While the biochemical and biophysical properties of NOD26 are well established, mRNA expression and genetic studies of NOD26 are lacking. Hence, the aim of the present study was to solve the long-standing question of nodule-specific expression and detailed expression pattern of NOD26 within the nodule. Besides, we were interested in exploring the hypothetical role of NOD26 in nitrogen-fixing symbiosis in planta by using the model legume species $M$. truncatula.

\section{Results And Discussion}

To characterize the function of both M. truncatula NOD26 copies (MtNOD26a and MtNOD26b) in terms of water transport activities, their cRNAs were individually expressed in Xenopus oocytes. In these assays, using 1:5 diluted ND96 solution (200 mOsm kg${ }^{-1}$ ), both MtNOD26a and MtNOD26b induced oocyte swelling (Fig. 2a). Specifically, the water permeability of these proteins was similar to each other and significantly higher than the water-injected oocytes (around three- to five-fold when 2.5 and $5 \mathrm{ng}$ cRNAs were used, respectively) (Fig. 2a). This indicates that both MtNOD26a and MtNOD26b are capable of increasing membrane permeability for water. As expected due to their evolutionary equivalence (Frare, et al. 2018) and extreme similarity (92\%), MtNOD26a and MtNOD26b display a conserved phosphorylation site at Ser262 (Fig. S1). This residue has been shown to be involved in stimulating water transport by soybean NOD26 (GmNOD26) (Guenther, et al. 2003). This observation suggests that the activities of MtNOD26a and MtNOD26b can be post-transcriptionally controlled, as shown for GmNOD26. In addition, the heterologous expression of $M$. truncatula and soybean NOD26 copies completely restores the ability of an ammonia uptake-defective strain (JW0441) to grow under nitrogen-limiting conditions at low pH (5.5) (Fig. 2b). This provides additional evidence supporting a role for NOD26 as ammonia transporter in acidic environments such as the symbiosome space of nitrogen-fixing plant cells (Pierre, et al. 2013). Taken together, our findings indicate that MtNOD26a and MtNOD26b are multifunctional channels.

Under the premise that MtNOD26a and MtNOD26b are specific pieces of the nitrogen-fixing symbiosis, we studied the expression of these genes at the mRNA level by using independent approaches. The initial examination of the expression of MtNOD26a and MtNOD26b by using microarray data suggests that their transcription is specifically highly stimulated in the nodule (Frare, et al. 2018). However, $M$. truncatula chip probes do not distinguish precisely between the two highly similar NOD26 copies. In this context, we quantified the accumulation of MtNOD26a and MtNOD26b transcripts in M. truncatula A17 leaves, shoots, nodules, roots and crowns by reverse transcription quantitative PCR (RT-qPCR), using 
primers that specifically target to differential non-coding 3'-UTR regions (Fig. S2). MtNOD26a and MtNOD26b transcripts highly accumulate in nodules but a few transcripts are also detected in roots at low levels (Fig. 2c). This result confirms that these genes are specifically upregulated in the nodule and is consistent with the notion that they emerged from an ancestral AQP expressed in roots (Frare, et al. 2018). This RT-qPCR also provides evidence that, in addition to its post-translational regulation (Guenther, et al. 2003). Together, these observations pinpoint the need for a finely-tuned activity of these multifunctional channels.

To provide independent evidence supporting a nodule-specific expression pattern of $M$. truncatula NOD26 copies and to analyze their expression in different plant stages and nodule zones, we produced stable transgenic plants expressing the GUS reporter under the control of the native promoters of MtNOD26a and MtNOD26b. Transcriptional activity elicited by the NOD26 promoters was observed at different nodule stages (Fig. 3a-f), but not in other localizations during different development stages such as seedlings (Fig. 3g-i) and adult plants (Fig. 3j-I), except for faint background activities of the MtNOD26b promoter fusion in adult roots (Fig. 3l). During nodule development, MtNOD26a and MtNOD26b expression increased, and MtNOD26b transcripts accumulated to higher levels than those of MtNOD26a (Fig. 3c-f). These findings show that the induction of the nodule-specific genes MtNOD26a and MtNOD26b accompanies nodule development and that their transcriptional activity and/or stability are not completely conserved, a fact that has important functional and evolutionary implications. The hypothesis of NOD26 origins and expansion in the nitrogen-fixing clade of angiosperms predicts that there should be intermediate stages between the ancestral nitrogen-fixing species containing two active NOD26 copies and their descendent plants exhibiting only one active copy (e.g. soybean, common bean, Lotus japonicus, Arachis ipaensis and Parasponia andersonii) (Frare, et al. 2018). Consequently, the differential expression levels of $M$. truncatula NOD26 copies here described can represent this intermediate stage, where MtNOD26a is evolving to an inactive gene.

Using these genetic constructs (pMtNOD26a-GUS and pMtNOD26b-GUS), the activity of GUS was found in different nodule tissues, including meristematic (I), pre-infection (II), infection (IZ) and nitrogen fixation (III) regions (Fig. 4a). In both transgenic plants, the strongest GUS staining was found in the fixation zone (Fig. 4a). These expression distributions within the nodule match the transcription data obtained by laser capture micro-dissection coupled to RNA sequencing (Roux, et al. 2014), where the expression of MtNOD26a and pMtNOD26b increases gradually from the bacteria-free meristematic region to the nitrogen-fixation zone (Fig. 4b). These results indicate that MtNOD26a and MtNOD26b behave as symbiotic genes and provide additional evidences of their functional equivalence. The functional equivalence between MtNOD26a and MtNOD26b in terms of transport activity and expression pattern could explain the common silencing of a single NOD26 copy observed in current nitrogen-fixing plants (Frare, et al. 2018), because the long-term maintenance of tandem paralogs needs the occurrence of neofunctionalization and/or subfunctionalization processes (Conant and Wolfe 2008). For example, we have previously described the long-term maintenance of the tandem-repeat AQPs NIP4;1 and NIP4;2 (critical components for pollen development and pollination) in flowering plants through modifications in 
their expression pattern, where each paralog probably retains a subset of original ancient function (Di Giorgio, et al. 2016).

To better understand the role of MtNOD26a and MtNOD26b in M. truncatula, the transposable element from the Nicotiana tabacum (Tnt1) mutant lines NF10538 (L26a) and NF6523 (L26b) were obtained from the Noble Foundation insertion mutant library (d'Erfurth, et al. 2003; Tadege, et al. 2008). According to the genome walking data, the 5kb-Tnt1 insertions are located in exon 2 of MtNOD26a and in intron 2 of MtNOD26b (Fig. 5a), and no wild-type alleles of these genes were detected in the homozygous mutant lines L26a and L26b (Fig. 5a). These knockout homozygous insertional mutant lines lacking NOD26 proteins allowed us to answer the long-standing question of whether these prominent nodulins are required for symbiotic nitrogen fixation. To begin exploring this open question, we selected a moderate nitrogen-limiting condition (soil:vermiculite:perlite 1:1:1 v/v, irrigated with a minimal medium without nitrogen), where uninoculated wild-type plants had decreases of $33 \%$ in nitrogen content with respect to wild-type plants inoculated with rhizobia (Fig. S3). Under nitrogen deficiency, the mutant lines L26a and L26b exhibited the typical symptoms of nitrogen limitation, including stunted growth and yellow chlorotic leaves (Fig. 5b), compared to the wild-type plants, a fact reflected in significant decreases in the greenness index (Fig. 5c). Consistent with their nodule-specific expression pattern, MtNOD26a and MtNOD26b were not required for plant growth under nonrestrictive nutrient (100\% soil) conditions (Fig. S4). In accordance with their symptoms of nitrogen deficit, the mutant lines L26a and L26b displayed the same aberrant nodulation phenotype (Fig. $5 \mathrm{~d}$ ), consisting of higher nodules (Fig. 5e), which are frequently clustered together (Fig. $5 f$ ) in an unaltered number (Fig. 5g). In addition to providing additional evidences of the functional equivalence between MtNOD26a and MtNOD26b, these results indicate that the loss of function of these two NOD26 genes of $M$. truncatula triggers a loss of nodule identity, consequently leading to nitrogen fixation failure.

Under symbiotic conditions in which plants were completely dependent upon rhizobia to fix nitrogen, specifically in minimal medium without a nitrogen source in axenic growth conditions, both the uninoculated and inoculated mutant lines L26a and L26b displayed the same symptoms of extreme nitrogen limitation, including severely yellow chlorotic leaves (Fig. 6a) and stunted growth (Fig. 6a-b), which reflect the nonsymbiotic phenotype of the uninoculated wild-type plants. These results allow concluding that the symbiotic NOD26 proteins have evolved a symbiosis-specific set of functions that are required for symbiotic nitrogen fixation.

The biogenesis of the SM results in the acquisition of a large number of specific transport activities related to the establishment and the maintenance of the symbiosis (Cai, et al. 2018; Magne, et al. 2018; Mergaert, et al. 2020; Pislariu, et al. 2019). Generally, the massive activation of nodule-specific genes during symbiotic cell differentiation is driven by endoreduplication-dependent epigenetic changes (Nagymihály, et al. 2017). In addition to their functions in the emergence of critical novelties for rapid plant diversification (Di Giorgio, et al. 2016; Perez Di Giorgio, et al. 2014), tandem duplications are a rudimentary but extremely effective strategy to maximize the transcription of genes playing physiological and structural essential roles (Panchy, et al. 2016). This is mainly related to the gene dosage (number of 
active NOD26 copies), but also to the fact that the expression of tandem gene copies is normally more than twofold higher than that of the ancestral single gene (Loehlin and Carroll 2016). We have previously shown that the emergence of NOD26, the most abundant nodulin, in the nitrogen-fixing clade of angiosperms, as well as its expansion in different nitrogen-fixing plant lineages, occurred by both block and tandem duplications (Frare, et al. 2018). One of the most important predictions derived from this evolutionary reconstruction is the relevance of the gene dosage in the initial stabilization of the symbiotic nitrogen fixation mechanism in the legume family (Frare, et al. 2018). Furthermore, in accordance with this prediction, we have shown that the presence of both nodule-specific NOD26 copies of the model legume species $M$. truncatula is required for symbiotic nitrogen fixation, empirically recapitulating the crucial contribution of NOD26 copies for effective symbiotic nitrogen fixation in ancestral nitrogen-fixing species.

The metabolic reconstruction of the origin of the nitrogen-fixation precursor in angiosperms exposed the probable ancestral non-symbiotic role of NOD26 in osmotic balance and ammonia detoxification in anoxic roots (Frare, et al. 2018). Importantly, numerous empirical evidences suggest the possible role of NOD26 in the controlled movement of ammonia and water through the SM in nitrogen-fixing plant cells (Dean, et al. 1999; Fortin, et al. 1987; Frare, et al. 2020; Guenther, et al. 2003; Hwang, et al. 2010; Masalkar, et al. 2010; Rivers, et al. 1997; Weaver, et al. 1991). Similarly, mammals have the multifunctional channel aquaporin-8 (AQP8), which is localized in mitochondria of liver cells and is responsible for the efflux of water and ammonia generated by oxidative phosphorylation and acidosis, respectively (Fig. S7) (Ikaga, et al. 2015; Molinas, et al. 2012; Soria, et al. 2010). These genetic studies have demonstrated that AQP8 has crucial roles in mammals, but similar data for its putative analogous plant protein NOD26 are still lacking (Mergaert, et al. 2020). Our physiological studies of the mutant lines L26a and L26b revealed the crucial contribution of NOD26 to effective symbiotic nitrogen fixation. Therefore, this work represents the first demonstration of the essential role of NOD26 in this symbiotic process and helps to close the gap in the understanding of AQP functions in plants vs those in animals.

\section{Experimental Procedures}

\section{Plant material and growth conditions}

Wild-type Medicago truncatula A17 (Hoffmann, et al. 1997) and its corresponding mutants NF10538 (L26a) and NF6523 (L26b), obtained from the Noble Foundation (https://medicago-mutant.noble.org), were cultivated in soil (with an average nitrogen content of $20 \mathrm{mg} \mathrm{kg}^{-1}$ ) or in a mixture of soil, vermiculite and perlite $(1: 1: 1, \mathrm{v} / \mathrm{v})$, and irrigated with the minimal medium INTA13 without nitrogen (Setten, et al. 2013) in 1-L pots under non-gnotobiotic conditions or on nitrogen-free agar (INTA13) in 0.1-L glass tubes under axenic conditions. The regenerative clone C2-3 (García, et al. 2014) and its derived transgenic plants pMtNOD26a-GUS and pMtNOD26b-GUS (this work) were cultivated in vermiculite irrigated with INTA13 without nitrogen under non-gnotobiotic conditions. Seeds were surface-sterilized in sulphuric acid for 8 min and treated or not with $1 \times 10^{6}$ colony-forming units per seed of the model strain Sinorhizobium meliloti 2011 (Galibert, et al. 2001) 14 days post-sowing. Plants were grown in a controlled environmental 
chamber with a $16 / 8$-h light/dark cycle, at $23^{\circ} \mathrm{C}$, relative humidity of $50 \%$, and $120 \mu \mathrm{mol} \mathrm{m}^{-2} \mathrm{sec}^{-1}$ light intensity.

\section{Quantitative RT-PCR (RT-qPCR) assays}

cDNA total RNA was extracted from each tissue, using an RNeasy Plant Mini Kit (QIAGEN Cat 74903, Germany), treated with RQ1 RNase-Free Dnase (Promega) and used as a template to generate first-strand cDNA synthesis, using RevertAid Reverse Transcriptase (ThermoFisher) with random primers (Promega) and dNTPs (Invitrogen). Then, $2 \mu \mathrm{g}$ total RNA was used for each reaction. The relative mRNA abundance was evaluated via quantitative reverse transcription PCR (RT-qPCR) in a total reaction volume of $20 \mu \mathrm{L}$ using FastStart Essential DNA Green Master (Roche) on a LightCycler96 Real-Time PCR System (Roche) with $1 \mu \mathrm{M}$ of each specific sense and anti-sense primer (Fig. S5). Three independent biological replicates of each sample and three technical replicates of each biological replicate were performed and the mean values were considered for further calculations. The normalized expression of MtNOD26a and MtNOD26b was calculated using M. truncatula ubiquitin as the endogenous reference gene.

\section{Heterologous expression assays}

cDNAs of MtNOD26a and MtNOD26b were amplified by PCR from nodule cDNA, cloned into pGEM-easy vector (Fig. S5), sequenced, and subcloned in a pT7Ts-derived vector carrying the $5^{\prime}$ and $3^{\prime}$ untranslated sequences of the Xenopus $\beta$-globin gene (Alleva, et al. 2010). cRNAs were synthesized using T7 RNA polymerase and purified following the manufacturer's instructions (mMESSAGE mMACHINET7 High Yield Capped RNA Transcription Kit, Ambion, Austin, USA). cRNAs were suspended in water and diluted to a


solution (Alleva, et al. 2010) supplemented with $1 \mu \mathrm{g} \mathrm{mL}^{-1}$ gentamicin at $18^{\circ} \mathrm{C}$. Defolliculated oocytes were microinjected with $50 \mathrm{~nL}$ of in vitro cRNAs (2.5 or $5 \mathrm{ng}$ ). Distilled water was used as negative control. Typically, 3 days after the injection, single oocytes were individually transferred from the ND96 solution (200 mOsm kg-1) to a ND96 solution diluted 1:5 with water (final osmolarity $40 \mathrm{mOsm} \mathrm{kg}^{-1}$ ). Changes in cell volume were monitored and videocaptured as described previously (Soto, et al. 2008). Complementation of the ammonia uptake-defective strain JW0441 with plasmids pGmNOD26a and pMtNOD26a, which contain the soybean and $M$. truncatula NOD26a genes within the expression vector pSEVA4413 (Fig. S5), was determined in solid M9 minimal medium supplemented with $0.1 \mathrm{mM} \mathrm{NH}_{4} \mathrm{Cl}$ at pH 5.5, as previously described (Frare, et al. 2020).

\section{Production and characterization of transcriptional fusion lines}

Genomic DNA of $M$. truncatula plants was isolated from leaf tissue by using the DNeasy plant mini kit (Cat.\#69104, Qiagen). For the constructions of the binary vectors pMtNOD26a-GUS and pMtNOD26bGUS, two-kb sections upstream of the MtNOD26a and MtNOD26b start codon were amplified (Fig. S5), cloned in the pGEM-easy vector, sequenced and subcloned in the binary vector pBI121 (García, et al. 2014). We produced stable transformants that carry the pMtNOD26a-GUS and pMtNOD26b-GUS fusions 
by using wild-type regenerative clone C2-3 as previously (Jozefkowicz, et al. 2018; Pascuan, et al. 2020). Kanamycin-tolerant plants derived from different calluses were considered independent transgenic events. These pMtNOD26a-GUS- and pMtNOD26b-GUS-transformed events were individually crossed manually with wild-type plants by using transgenic parental plants as pollen donors. The progenies of pMtNOD26a-GUS and pMtNOD26b-GUS were analyzed by histochemical detection of GUS activity (Kelemen, et al. 2002). The differential expression of transcriptional fusions (Fig. 3c-f) was observed in the 98 and 54 independent events produced by pMtNOD26a-GUS and pMtNOD26b-GUS, respectively. For histological analysis, nodules were fixed overnight at $4^{\circ} \mathrm{C}$ in $4 \%$ paraformaldehyde with $0.1 \mathrm{M}$ phosphate buffered saline (PBS) (pH 7.2), dehydrated in a graded ethanol and xylene series, and embedded in paraffin. Sections $(10 \mu \mathrm{m})$ were mounted on glass slides. Images were obtained by light microscopy with an Axioskope 2 microscope (Carl Zeiss, Jena, Germany) and captured with an EOS 1000D camera (Canon, Tokyo, Japan).

\section{Identification and characterization of mutant lines}

As described in the Noble Foundation insertion mutant library, we confirmed that the Tnt1 flanking sequences from the insertional lines L26a (NF10538) and L26b (NF6523) match completely and distinctively to Medtr8g087710 and Medtr8g087720, respectively (Fig. S6). This was confirmed by using the Mapper in Geneious Prime (www.geneious.com) with standard parameters. Genomic DNA for genotyping analysis was isolated as described previously (Soto, et al. 2010). The insertional mutants were genotyped by PCR (Fig. S5). Neither MtNOD26a nor MtNOD26b transcripts were detected by the RT-

qPCR assays in the mutant lines L26a and L26b, respectively (Fig. S5). The greenness index of wild-type and mutant plants was analyzed using CCM-200-Opti-Sciences by measuring the light transmittance between red $(650 \mathrm{~nm})$ and near-infrared $(940 \mathrm{~nm})$ chlorophyll absorption ranges (Torres-Dorante, et al. 2016). Total nitrogen content in leaves was measured by the Kjeldahl method, as previously (Fox, et al. 2016).

\section{Declarations}

\section{Acknowledgements.}

This work was supported by FONCyT-Argentina, Grants PICT-2015-0090 and PICT-2017-0484 to GS, and by CONICET-Argentina, Grant PIP-2014-2106 to KA, NA and GS.

\section{References}

Alleva K, Marquez M, Villarreal N, Mut P, Bustamante C, Bellati J, Martínez G, Civello M, Amodeo G (2010) Cloning, functional characterization, and co-expression studies of a novel aquaporin (FaPIP2;1) of strawberry fruit. Journal of Experimental Botany 61:3935-3945

Beamer Z, Routray P, Choi W-G, Spangler MK, Lokdarshi A, Roberts DM (2020) The Arabidopsis thaliana NIP2;1 Lactic Acid Channel promotes Plant Survival Under Low Oxygen Stress. bioRxiv 
Blumwald E, Fortin MG, Rea PA, Verma DP, Poole RJ (1985) Presence of Host-Plasma Membrane Type HATPase in the Membrane Envelope Enclosing the Bacteroids in Soybean Root Nodules. Plant physiology 78:665-672

Cai J, Zhang L-Y, Liu W, Tian Y, Xiong J-S, Wang Y-H, Li R-J, Li H-M, Wen J, Mysore KS, Boller T, Xie Z-P, Staehelin C (2018) Role of the Nod Factor Hydrolase MtNFH1 in Regulating Nod Factor Levels during Rhizobial Infection and in Mature Nodules of Medicago truncatula. The Plant Cell 30:397-414

Clarke VC, Loughlin PC, Gavrin A, Chen C, Brear EM, Day DA, Smith PM (2015) Proteomic analysis of the soybean symbiosome identifies new symbiotic proteins. Mol Cell Proteomics 14:1301-1322

Conant GC, Wolfe KH (2008) Turning a hobby into a job: how duplicated genes find new functions. Nature reviews Genetics 9:938-950

d'Erfurth I, Cosson V, Eschstruth A, Lucas H, Kondorosi A, Ratet P (2003) Efficient transposition of the Tnt1 tobacco retrotransposon in the model legume Medicago truncatula. The Plant journal : for cell and molecular biology 34:95-106

Dean RM, Rivers RL, Zeidel ML, Roberts DM (1999) Purification and functional reconstitution of soybean nodulin 26. An aquaporin with water and glycerol transport properties. Biochemistry 38:347-353

Di Giorgio JAP, Bienert GP, Ayub ND, Yaneff A, Barberini ML, Mecchia MA, Amodeo G, Soto GC, Muschietti JP (2016) Pollen-Specific Aquaporins NIP4;1 and NIP4;2 Are Required for Pollen Development and Pollination in Arabidopsis thaliana. The Plant Cell 28:1053-1077

diCenzo GC, Tesi M, Pfau T, Mengoni A, Fondi M (2020) Genome-scale metabolic reconstruction of the symbiosis between a leguminous plant and a nitrogen-fixing bacterium. Nat Commun 11:2574

Fortin MG, Morrison NA, Verma DP (1987) Nodulin-26, a peribacteroid membrane nodulin is expressed independently of the development of the peribacteroid compartment. Nucleic Acids Res 15:813-824

Fox AR, Soto G, Valverde C, Russo D, Lagares Jr A, Zorreguieta Á, Alleva K, Pascuan C, Frare R, MercadoBlanco J, Dixon R, Ayub ND (2016) Major cereal crops benefit from biological nitrogen fixation when inoculated with the nitrogen-fixing bacterium Pseudomonas protegens Pf-5 X940. Environmental Microbiology 18:3522-3534

Frare R, Ayub N, Alleva K, Soto G (2018) The Ammonium Channel NOD26 is the Evolutionary Innovation that Drives the Emergence, Consolidation, and Dissemination of Nitrogen-Fixing Symbiosis in Angiosperms. J Mol Evol 86:554-565

Frare R, Stritzler M, Pascuan C, Liebrenz K, Galindo-Sotomonte L, Soto L, Nikel PI, Ayub N (2020) Elimination of GInKAmtB affects serine biosynthesis and improves growth and stress tolerance of 
Escherichia coli under nutrient-rich conditions. FEMS Microbiology Letters accepted manuscript 10.1093/femsle/fnaa1197

Galibert F, Finan TM, Long SR, Batut J (2001) The composite genome of the legume symbiont Sinorhizobium meliloti. Science (New York, NY) 293:668-672

García AN, Ayub ND, Fox AR, Gómez MC, Diéguez MJ, Pagano EM, Berini CA, Muschietti JP, Soto G (2014) Alfalfa snakin-1 prevents fungal colonization and probably coevolved with rhizobia. BMC Plant Biology $14: 248$

Guenther JF, Chanmanivone N, Galetovic MP, Wallace IS, Cobb JA, Roberts DM (2003) Phosphorylation of soybean nodulin 26 on serine 262 enhances water permeability and is regulated developmentally and by osmotic signals. Plant Cell 15:981-991

Hoffmann B, Trinh TH, Leung J, Kondorosi A, Kondorosi E (1997) A New Medicago truncatula Line with Superior in Vitro Regeneration, Transformation, and Symbiotic Properties Isolated Through Cell Culture Selection. Molecular Plant-Microbe Interactions ${ }^{\circledR}$ 10:307-315

Hwang JH, Ellingson SR, Roberts DM (2010) Ammonia permeability of the soybean nodulin 26 channel. FEBS Lett 584:4339-4343

Ikaga R, Namekata I, Kotiadis VN, Ogawa H, Duchen MR, Tanaka H, lida-Tanaka N (2015) Knockdown of aquaporin-8 induces mitochondrial dysfunction in 3T3-L1 cells. Biochemistry and biophysics reports 4:187-195

Jones KM, Kobayashi H, Davies BW, Taga ME, Walker GC (2007) How rhizobial symbionts invade plants: the Sinorhizobium-Medicago model. Nature Reviews Microbiology 5:619-633

Jozefkowicz C, Frare R, Fox R, Odorizzi A, Arolfo V, Pagano E, Basigalup D, Ayub N, Soto G (2018) Maximizing the expression of transgenic traits into elite alfalfa germplasm using a supertransgene configuration in heterozygous conditions. Theoretical and Applied Genetics 131:1111-1123

Kelemen Z, Mai A, Kapros T, Feher A, Gyorgyey J, Waterborg JH, Dudits D (2002) Transformation vector based on promoter and intron sequences of a replacement histone $\mathrm{H} 3$ gene. A tool for high, constitutive gene expression in plants. Transgenic Res 11:69-72

Loehlin DW, Carroll SB (2016) Expression of tandem gene duplicates is often greater than twofold. Proceedings of the National Academy of Sciences 113:5988-5992

Magne K, Couzigou J-M, Schiessl K, Liu S, George J, Zhukov V, Sahl L, Boyer F, lantcheva A, Mysore KS, Wen J, Citerne S, Oldroyd GED, Ratet P (2018) MtNODULE ROOT1 and MtNODULE ROOT2 Are Essential for Indeterminate Nodule Identity. Plant Physiology 178:295-316 
Masalkar P, Wallace IS, Hwang JH, Roberts DM (2010) Interaction of cytosolic glutamine synthetase of soybean root nodules with the C-terminal domain of the symbiosome membrane nodulin 26 aquaglyceroporin. J Biol Chem 285:23880-23888

Mergaert P, Kereszt A, Kondorosi E (2020) Gene Expression in Nitrogen-Fixing Symbiotic Nodule Cells in Medicago truncatula and Other Nodulating Plants. Plant Cell 32:42-68

Molinas SM, Trumper L, Marinelli RA (2012) Mitochondrial aquaporin-8 in renal proximal tubule cells: evidence for a role in the response to metabolic acidosis. American journal of physiology Renal physiology 303:F458-466

Nagymihály M, Veluchamy A, Györgypál Z, Ariel F, Jégu T, Benhamed M, Szücs A, Kereszt A, Mergaert P, Kondorosi É (2017) Ploidy-dependent changes in the epigenome of symbiotic cells correlate with specific patterns of gene expression. Proc Natl Acad Sci U S A 114:4543-4548

Niemietz CM, Tyerman SD (2000) Channel-mediated permeation of ammonia gas through the peribacteroid membrane of soybean nodules. FEBS Letters 465:110-114

Panchy N, Lehti-Shiu M, Shiu S-H (2016) Evolution of Gene Duplication in Plants. Plant physiology 171:2294-2316

Pascuan C, Bottero E, Kapros T, Ayub N, Soto G (2020) pBAR-H3.2, a native-optimized binary vector to bypass transgene silencing in alfalfa. Plant Cell Reports 39:683-685

Perez Di Giorgio J, Soto G, Alleva K, Jozefkowicz C, Amodeo G, Muschietti JP, Ayub ND (2014) Prediction of aquaporin function by integrating evolutionary and functional analyses. J Membr Biol 247:107-125

Pierre O, ENGLER G, HOPKINS J, BRAU F, BONCOMPAGNI E, HÉROUART D (2013) Peribacteroid space acidification: a marker of mature bacteroid functioning in Medicago truncatula nodules. Plant, Cell \& Environment 36:2059-2070

Pislariu Cl, Sinharoy S, Torres-Jerez I, Nakashima J, Blancaflor EB, Udvardi MK (2019) The NoduleSpecific PLAT Domain Protein NPD1 Is Required for Nitrogen-Fixing Symbiosis. Plant Physiology 180:1480-1497

Rivers RL, Dean RM, Chandy G, Hall JE, Roberts DM, Zeidel ML (1997) Functional analysis of nodulin 26, an aquaporin in soybean root nodule symbiosomes. J Biol Chem 272:16256-16261

Routray P, Masalkar PD, Roberts DM (2015) Nodulin Intrinsic Proteins: Facilitators of Water and Ammonia Transport across the Symbiosome Membrane. In: Biological Nitrogen Fixation. pp 695-704

Roux B, Rodde N, Jardinaud MF, Timmers T, Sauviac L, Cottret L, Carrère S, Sallet E, Courcelle E, Moreau S, Debellé F, Capela D, de Carvalho-Niebel F, Gouzy J, Bruand C, Gamas P (2014) An integrated analysis of 
plant and bacterial gene expression in symbiotic root nodules using laser-capture microdissection coupled to RNA sequencing. The Plant journal : for cell and molecular biology 77:817-837

Setten L, Soto G, Mozzicafreddo M, Fox AR, Lisi C, Cuccioloni M, Angeletti M, Pagano E, Díaz-Paleo A, Ayub ND (2013) Engineering Pseudomonas protegens Pf-5 for Nitrogen Fixation and its Application to Improve Plant Growth under Nitrogen-Deficient Conditions. PLOS ONE 8:e63666

Soria LR, Fanelli E, Altamura N, Svelto M, Marinelli RA, Calamita G (2010) Aquaporin-8-facilitated mitochondrial ammonia transport. Biochemical and biophysical research communications 393:217-221

Soto G, Alleva K, Mazzella MA, Amodeo G, Muschietti JP (2008) AtTIP1;3 and AtTIP5;1, the only highly expressed Arabidopsis pollen-specific aquaporins, transport water and urea. FEBS Letters 582:4077-4082

Soto G, Fox R, Ayub N, Alleva K, Guaimas F, Erijman EJ, Mazzella A, Amodeo G, Muschietti J (2010) TIP5;1 is an aquaporin specifically targeted to pollen mitochondria and is probably involved in nitrogen remobilization in Arabidopsis thaliana. The Plant journal : for cell and molecular biology 64:1038-1047

Tadege M, Wen J, He J, Tu H, Kwak Y, Eschstruth A, Cayrel A, Endre G, Zhao PX, Chabaud M, Ratet P, Mysore KS (2008) Large-scale insertional mutagenesis using the Tnt1 retrotransposon in the model legume Medicago truncatula. The Plant journal : for cell and molecular biology 54:335-347

Torres-Dorante L, Paredes-Melesio R, Link A, Lammel J (2016) A methodology to develop algorithms that predict nitrogen fertilizer needs in maize based on chlorophyll measurements: a case study in Central Mexico. The Journal of Agricultural Science 154:705-719

Weaver CD, Crombie B, Stacey G, Roberts DM (1991) Calcium-dependent phosphorylation of symbiosome membrane proteins from nitrogen-fixing soybean nodules : evidence for phosphorylation of nodulin-26. Plant Physiol 95:222-227

\section{Figures}


(a)

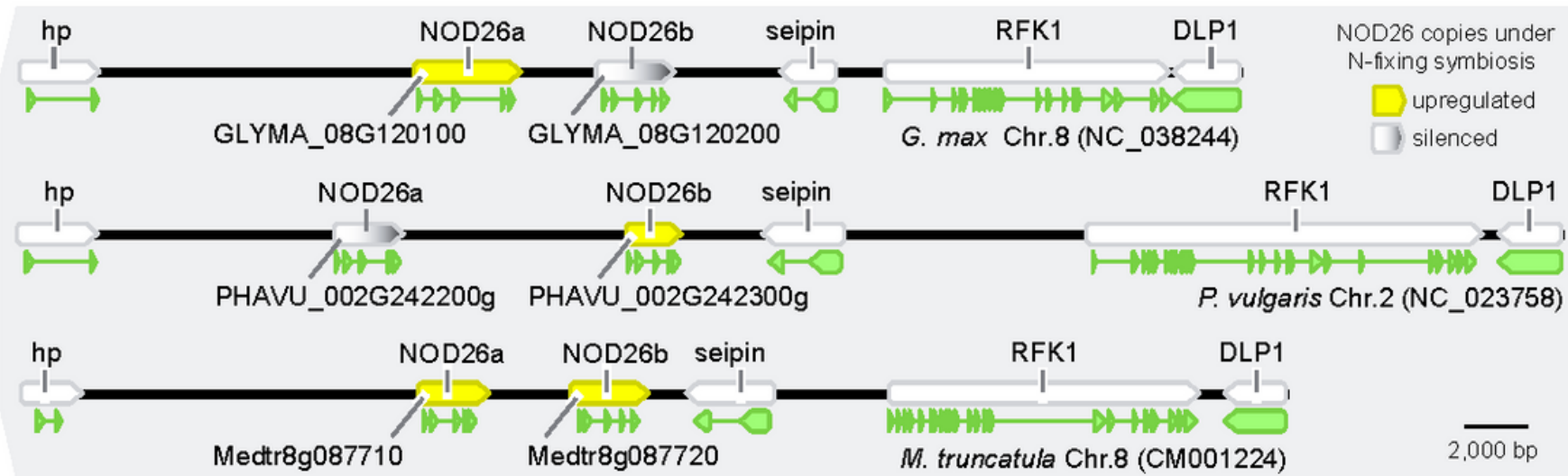

(b)

(c)


\section{Figure 1}

NOD26 tandem copies in legumes, their evolution and expression in symbiosis. (a) In the genome of nitrogen-fixing legumes, NOD26 is not a single-copy gene, but a tandem duplicated gene (NOD26a and NOD26b). This is both for crops (Glycine max = soybean and Phaseolus vulgaris = common bean) and model (Medicago truncatula) legume species. Microarray data suggest that most nitrogen-fixing species express only one copy of NOD26 (NOD26a or NOD26b) in nitrogen-fixing symbiosis (e.g. soybean and common bean), whereas M. truncatula seems to contain two active copies of NOD26. (b) NOD26a and NOD26b proteins clustered separately in the Neighbor Joining tree. (c) The most parsimonious explanation for these synteny and phylogenetic results and the microarray data is the occurrence of an ancestral nitrogen-fixing species containing two active NOD26 copies in symbiosis. This introductory picture is only a short revision of findings described previously (Frare, et al. 2018). 


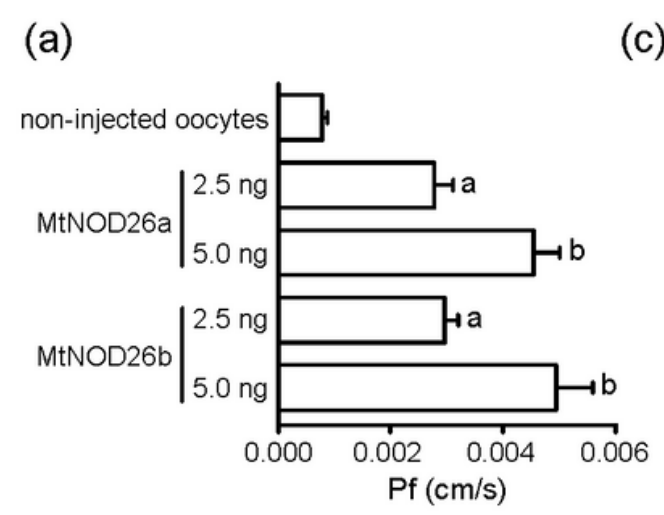

(b)

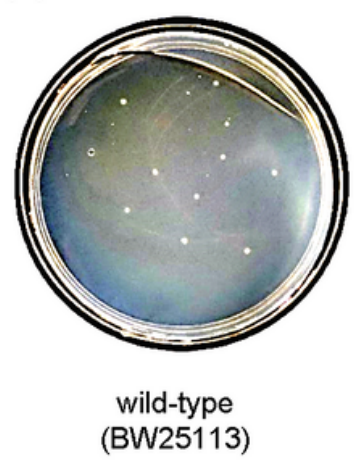
(BW25113)



(JW0441) (c)

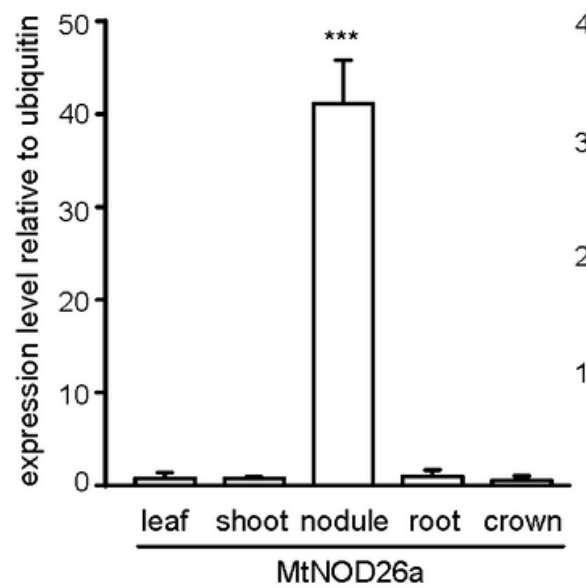

MtNOD26a

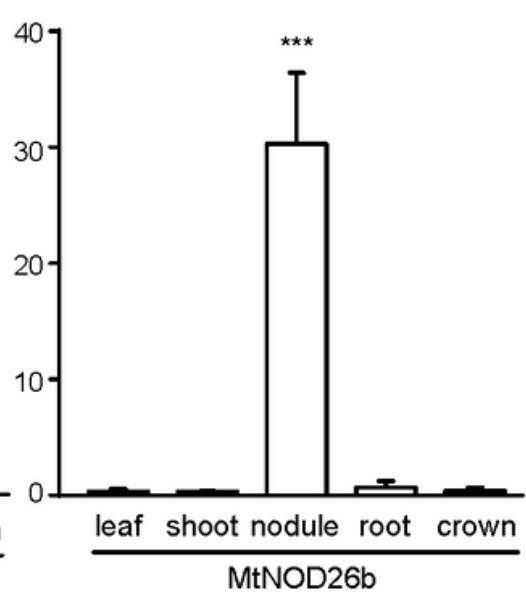

MtNOD26b

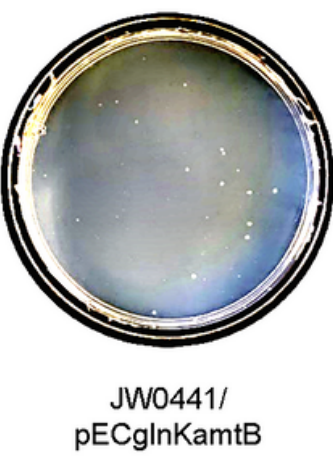

pECgInKamtB

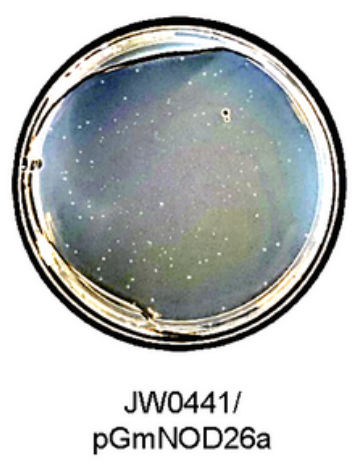

pGmNOD26a

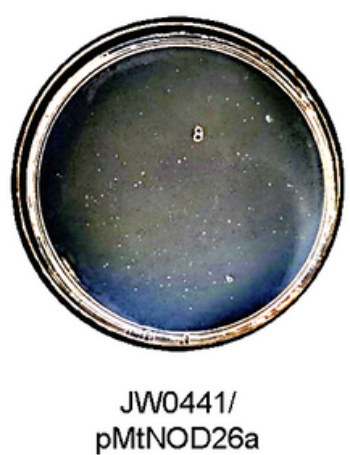

pMtNOD26a

\section{Figure 2}

Transport activities and expression patterns of MtNOD26a and MtNOD26b. (a) Both MtNOD26a and MtNOD26b show moderate levels of water permeability. Xenopus oocytes were injected either with water (negative control) or with MtNOD26a and MtNOD26b cRNAs and then tested for water permeability (Pf). Pf values were estimated from the rate of cell volume change upon hypo-osmotic shock. Independent experiments include different batches of oocytes (10-30 units for treatment) and different MtNOD26 in vitro transcriptions. All values are means + SEM $(n=3)$. Statistically significant differences are indicated by letters ( $p<0.05$, t-test). (b) When the ammonia uptake-defective strain JW0441, derived from wild-type strain BW25113, is complemented with its native ammonia transporter AmtB (pECglnKamtB) or with legume NOD26 genes (pGmNOD26a and pMtNOD26a, it is able to grow under nitrogen-limiting conditions $(0.1 \mathrm{mM} \mathrm{NH} 4 \mathrm{Cl})$ at low pH (5.5). Contrast was maximized to facilitate the visualization of colonies, which, in this minimal medium, are translucent. (c) MtNOD26a and MtNOD26b are specifically induced in nodules. The expression profiles of MtNOD26 genes in leaves, shoots, nodules, roots and crowns are relative to the housekeeping gene ubiquitin. Results represent means $\pm S E$ of three biological replicates ( 15 days post-inoculation (dpi)) and two technical replicates. Asterisks indicate significant differences compared with the nodule (***, $p<0.001$, one-way ANOVA). 




(g)
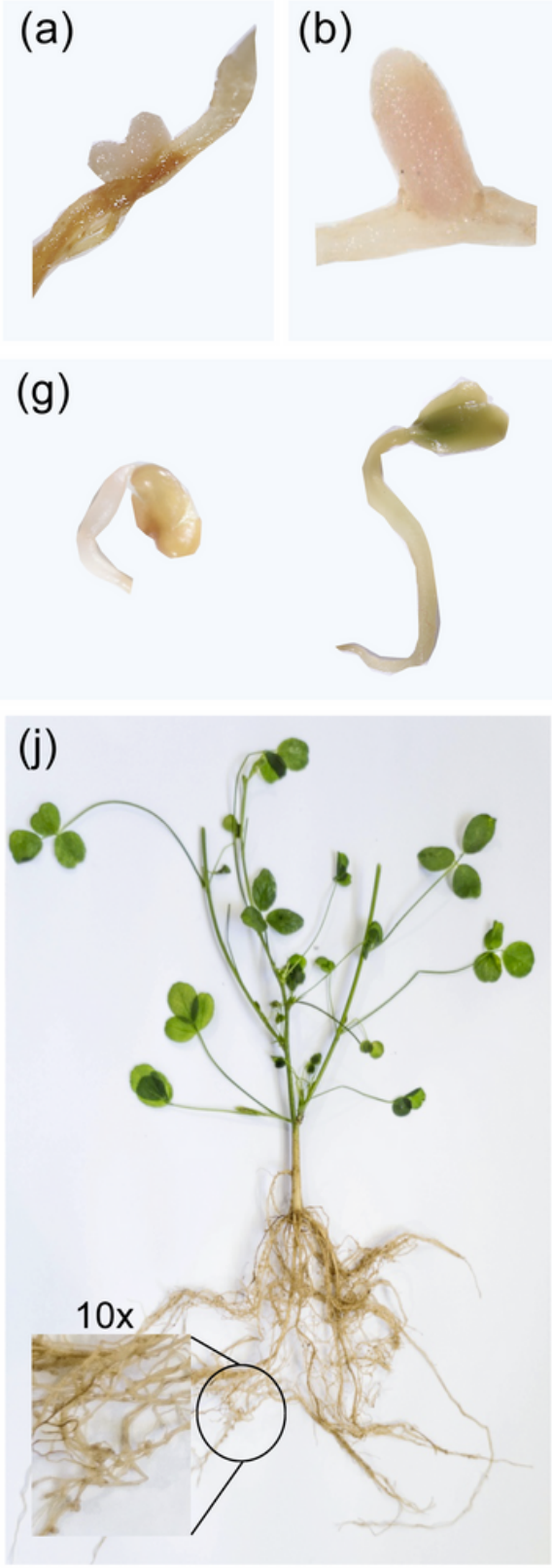

wild-type



(d)

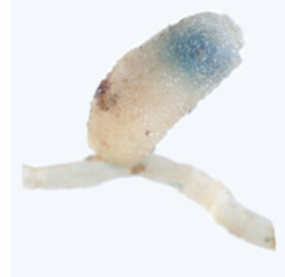

(h)

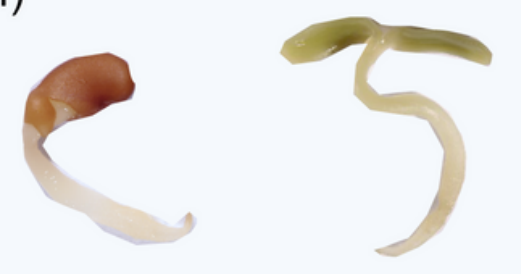

(k)

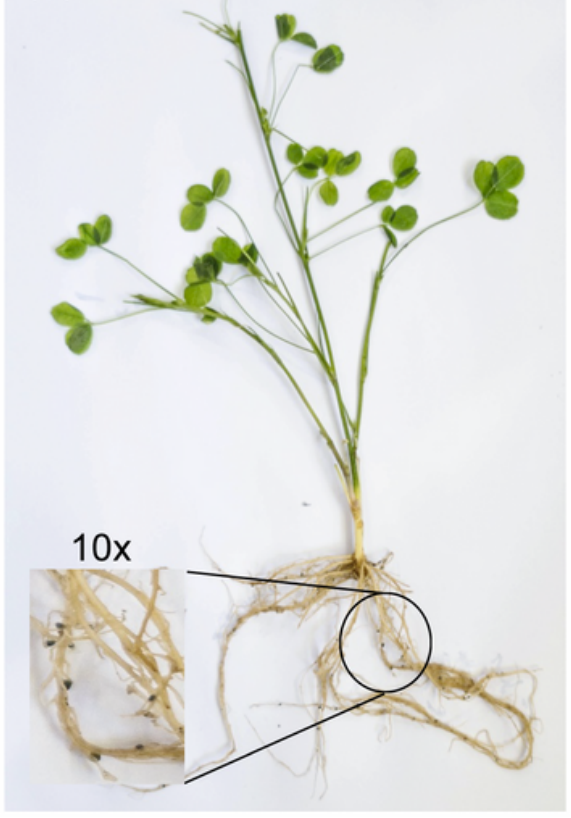

pMtNOD26a-GUS

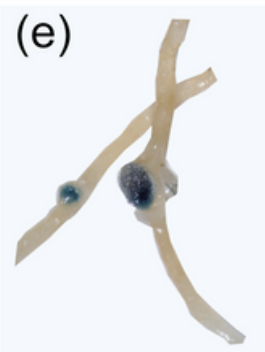

(f)

(i)
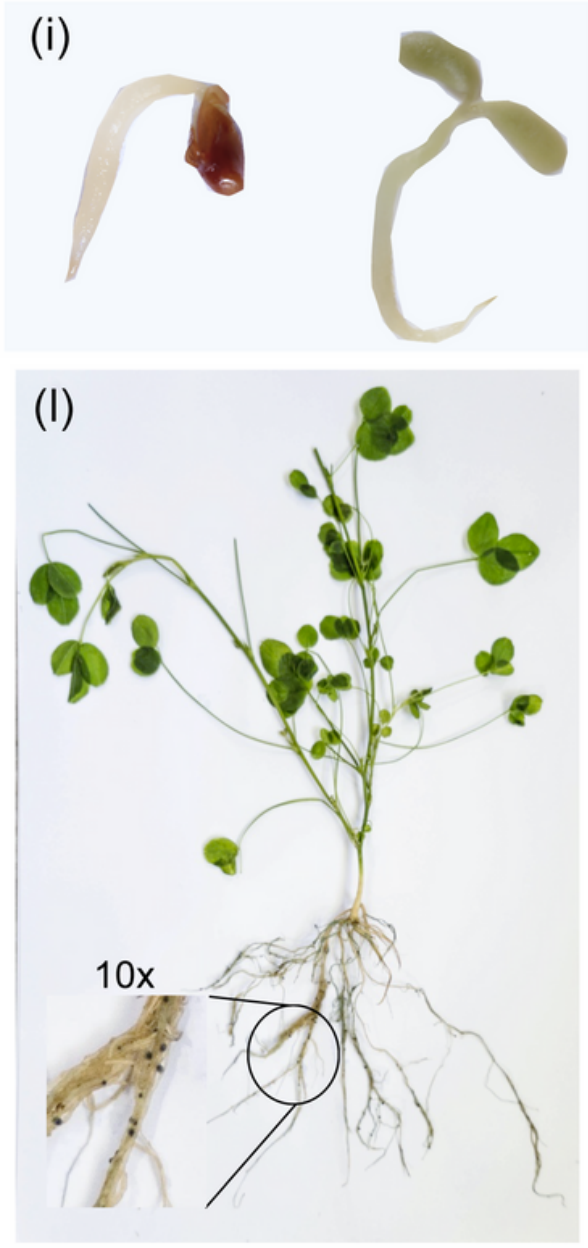

pMtNOD26b-GUS

\section{Figure 3}

Analysis of the expression of MtNOD26a and MtNOD26b at different nodule stages (early and mature), plant stages (seedlings and adults) and localizations (leaf, shoot, petiole, root and nodule) by using transgenic plants containing transcriptional fusions (pMtNOD26a-GUS and pMtNOD26b-GUS). This analysis shows the early and specific upregulation of the expression of MtNOD26 genes in nodules. Nodules inoculated with rhizobia at $10 \mathrm{dpi}(\mathrm{a}, \mathrm{c}$ and e) or $15 \mathrm{dpi}(\mathrm{b}, \mathrm{d}$ and $\mathrm{f}$ ). Seedlings of 1-3 days on the left and right panels, respectively ( $\mathrm{g}$ to $\mathrm{i}$ ) and adult plants of 3 months $(\mathrm{j}$ to $\mathrm{l}$ ). The figure shows representative results of at least fifty independent events obtained with pMtNOD26a and pMtNOD26b. 
(a)

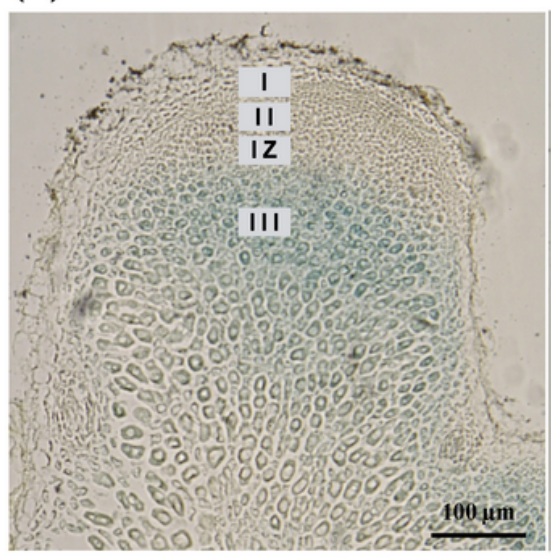

pMtNOD26a-GUS

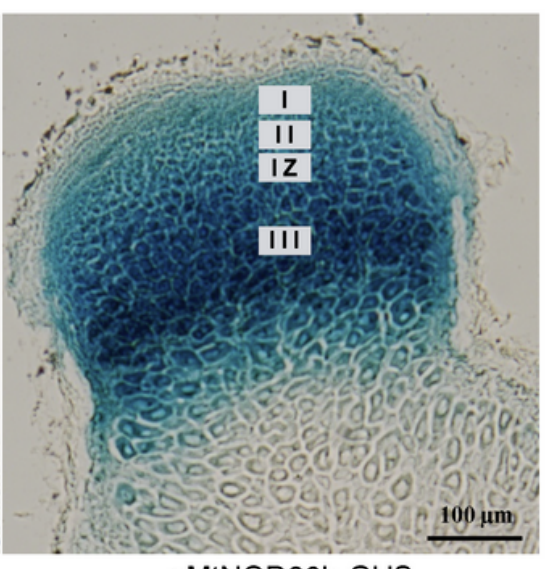

pMtNOD26b-GUS (b)

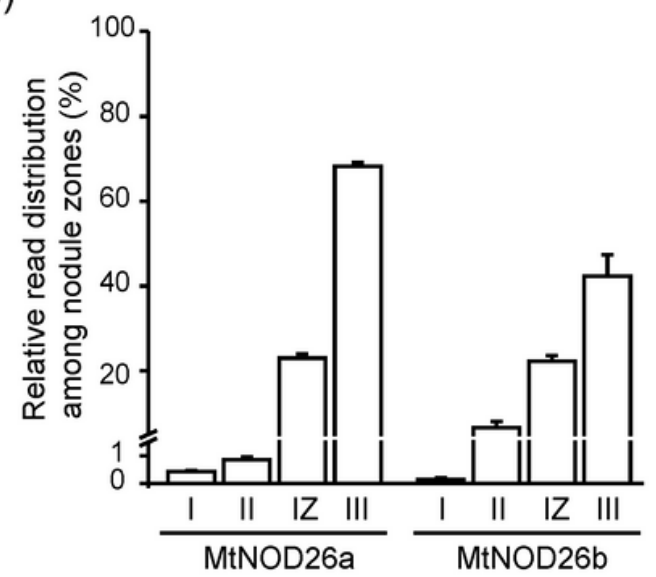

\section{Figure 4}

Analysis of the expression of MtNOD26a and MtNOD26b in diverse regions of the nodule (meristematic I, pre-infection II, infection IZ and nitrogen fixation III regions) at $15 \mathrm{dpi}$, by using transgenic plants pMtNOD26a-GUS and pMtNOD26b-GUS and RNA-seq data. This analysis shows the gradual increase in their expression from the bacteria-free meristematic region to the nitrogen-fixation zone. (a) Longitudinal medial light microscopic sections of nodules. The images are representative of at least 25 nodules from each construction. (b) Expression of M. truncatula MtNOD26 genes in nodules by laser-capture microdissection coupled to RNA sequencing. Data (mean $+\mathrm{SE}$ ) were obtained from Symbimics (iant.toulouse.inra.fr/symbimics/). 
(a)

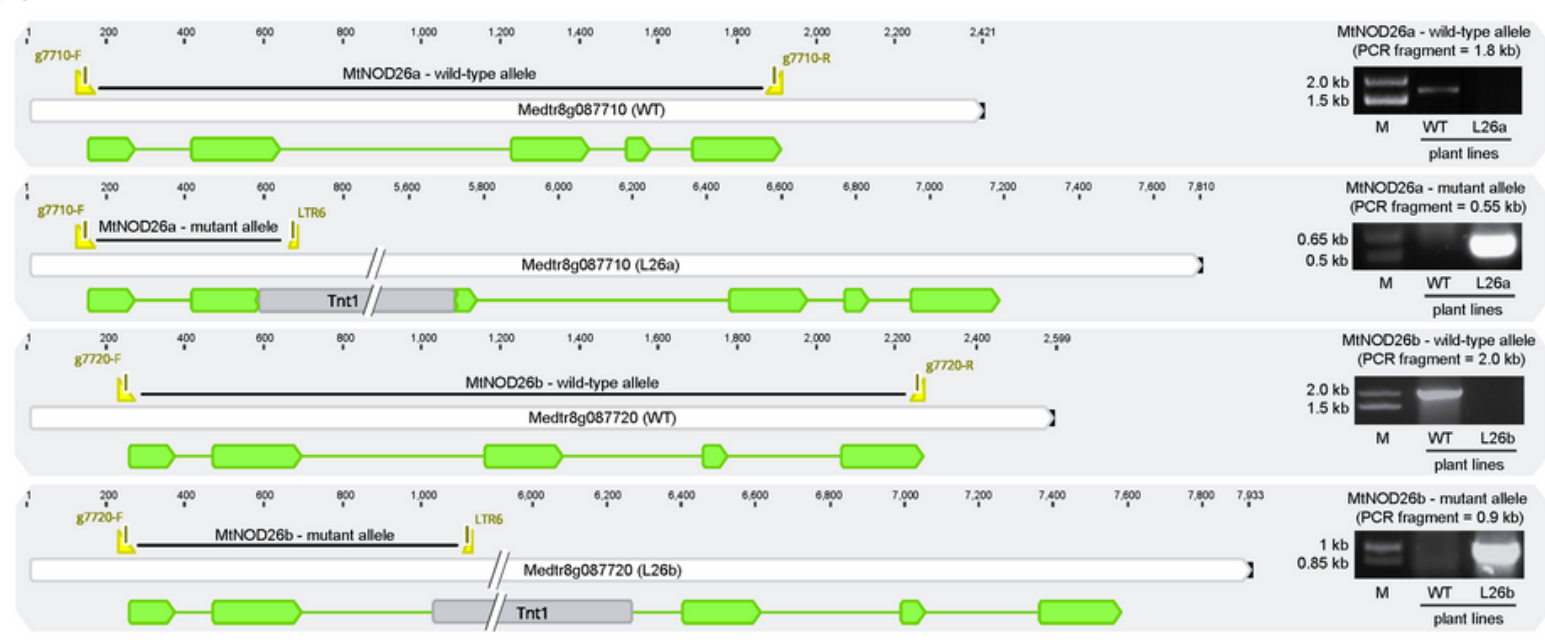

(b)

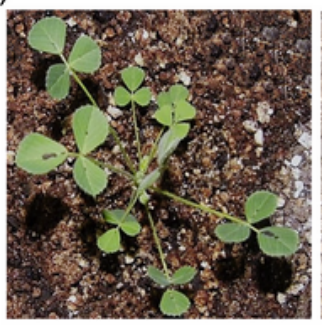

WT

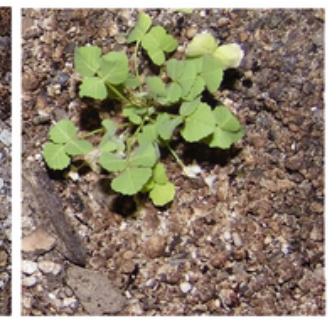

L26a

(c)

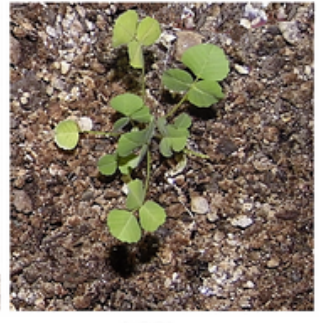

L26b

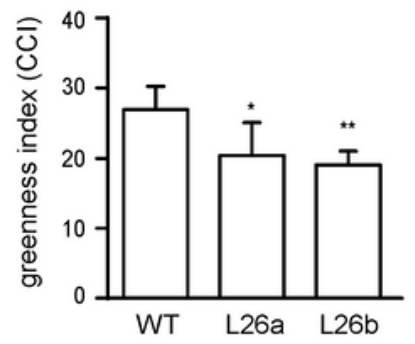

(d)
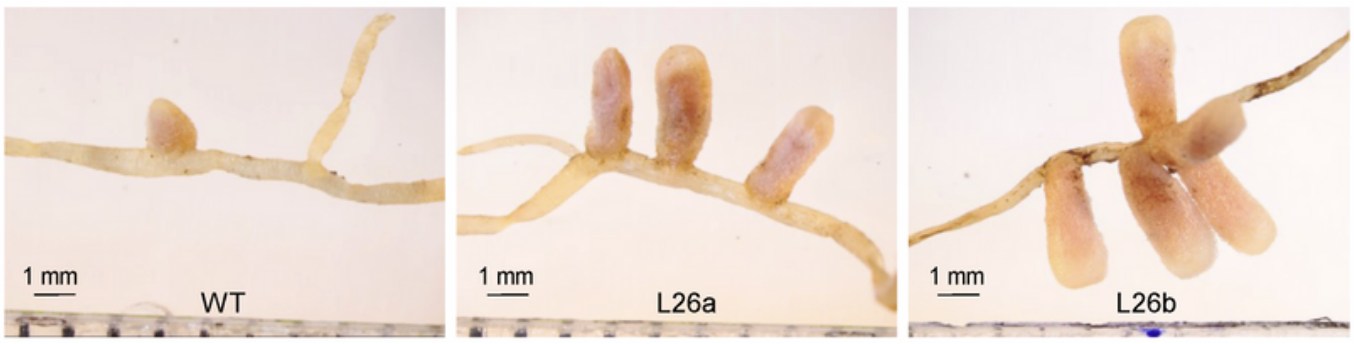

(e)

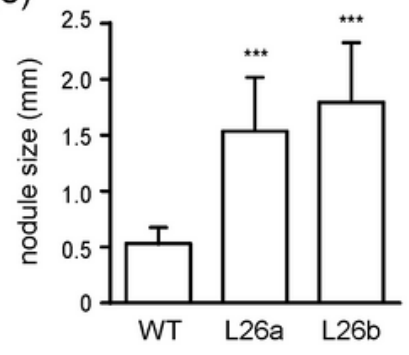

(f)

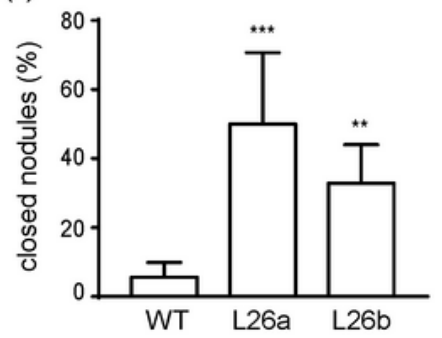

(g)

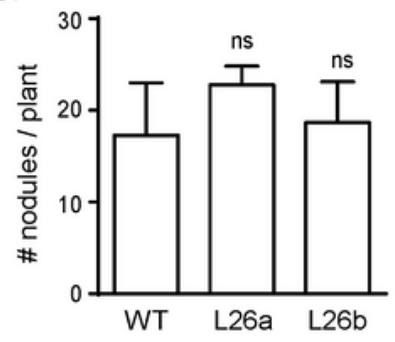

\section{Figure 5}

Characterization of the mutant lines L26a and Lb26 under nitrogen-limiting conditions. (a) Genotyping of the Tnt1 insertion mutant lines L26a (Medtr8g087710) and Lb26 (Medtr8g087710) in wild-type Medicago truncatula A17 background by PCR on genomic DNA. The black and green arrows represent mRNAs and exons, respectively, the gray boxes symbolize Tnt1 insertions, the yellow triangles indicate the primers used, and the black lines represent the alleles amplified in homozygous lines. (b) Under nitrogen 
deficiency, the Tnt1 insertion mutants for MtNOD26a (L26a) and MtNOD26b (L26b) show common symptoms of nitrogen limitation, such as reduced growth and yellow leaves, the latter of which is reflected in the reduction of their green color (c). L26a and L26b exhibit aberrant nodulation phenotype at $20 \mathrm{dpi}$ (d). Specifically, the mutant lines L26a and Lb26 produce higher and closer $(<5 \mathrm{~mm})$ nodules than their parental wild-type line A17. (e) The wild-type and mutant lines show similar nodule numbers. All values are means + SEM $(n=70)$. The statistical analysis was carried out with ANOVA followed by Dunnett's contrast test (ns not significant, ${ }^{*} p<0.05$, ${ }^{\star *} p<0.01, * \star \star p<0.001$ ). Statistical comparisons were made between wild-type plants and mutant lines. 
(a)

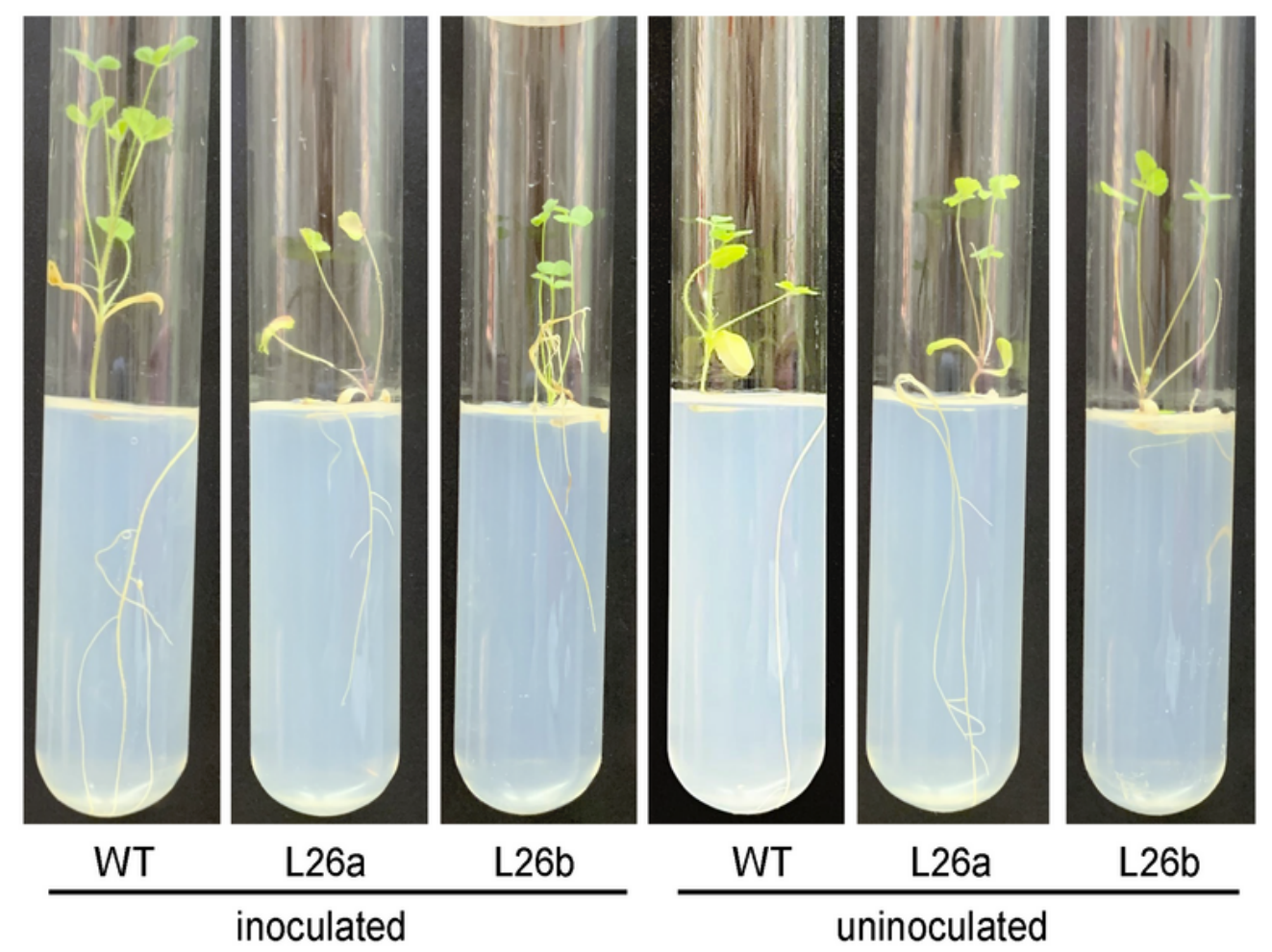

(b)

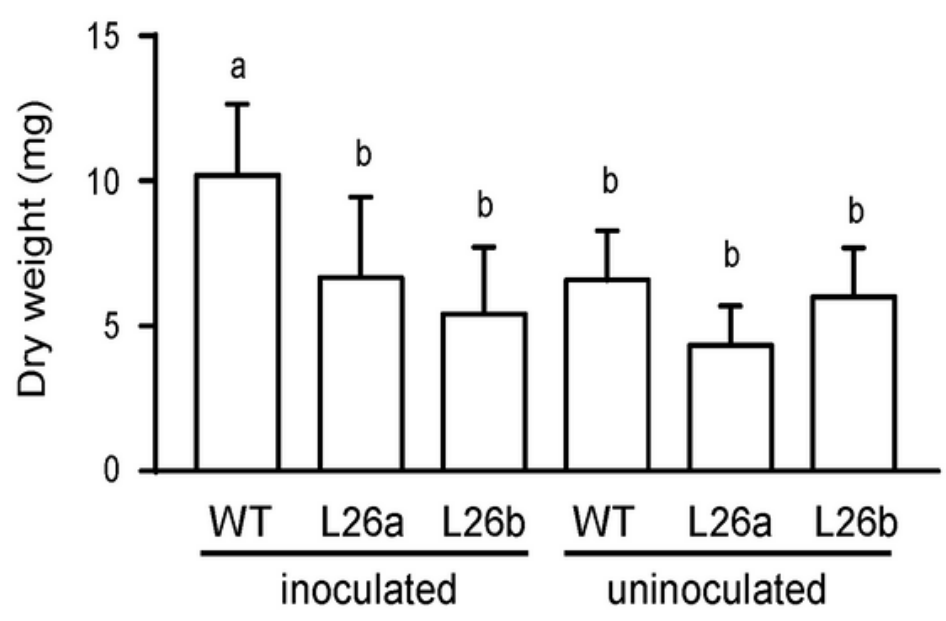

Figure 6

Characterization of the mutant lines L26a and Lb26 in a medium without nitrogen source. (a) In contrast to wild-type plants, mutant plants showed symptoms of extreme nitrogen limitation independently of the inoculation treatment at $45 \mathrm{dpi}$. (b) At this stage, the stunted growth of mutant plants can be measured as productivity. All values are means + SEM $(n=12)$. The statistical analysis was carried out with ANOVA followed by Dunnett's contrast test. Statistically significant differences are indicated by letters $(p<$ 
0.001). At $55 \mathrm{dpi}$, wild-type plants continued growth but uninoculated wild-type plants and all mutant plants died (data not shown).

\section{Supplementary Files}

This is a list of supplementary files associated with this preprint. Click to download.

- FigS1.pdf

- Figs2.pdf

- FigS3.pdf

- FigS4.pdf

- FigS5.pdf

- FigS6.pdf

- FigS7.pdf 